\title{
ABUNDANCE RATIOS IN COMPOSITE STELLAR POPULATIONS WITH SPECIAL EMPHASIS ON ELLIPTICAL GALAXIES
}

\author{
UTA FRITZE - VON ALVENSLEBEN \\ Universitätssternwarte Göttingen \\ Geismarlandstr. 11, 37083 Göttingen, Germany
}

\section{Introduction}

Abundance Ratios are observed in a wide variety of instances addressed in this volume: in Gas and Stars, Simple Stellar Populations (SSPs) like star clusters, and, finally, in Composite Stellar Populations (CSPs) like E/S0 galaxies (Bulges, spheroidal galaxies, Merger Remnants, etc.) on which the focus of this contribution will be. I'll briefly review the observational situation and the current interpretation and then cast some doubt on the latter from our own chemically consistent spectrophotometric modelling results.

\section{Observations and Standard Interpretation}

As defined in the Lick system, absorption line indices like Fe5270, Fe5335, $\mathrm{Mg}_{2}$, $\mathrm{Mgh}$, and many others have been measured by various groups on large numbers of early type galaxies and bulges. For the nuclei of giant field ellipticals Fe5270 and Fe5335 are seen to correlate with $\mathrm{Mg}_{2}$, but the slopes of Fe5270 and Fe5335 versus $\mathrm{Mg}_{2}$ are found to be rather shallow with considerable scatter, they do not follow the relation for globular clusters, and they are flatter for nuclei of different galaxies than within one galaxy (see e.g. Faber et al. 1992, Worthey et al. 1992, Davies et al. 1993). Gradients in $\mathrm{Mg}_{2}$ tend to be stronger for larger Es, gradients in Fe correlate with gradients in $\mathrm{Mg}_{2} \cdot \mathrm{Mg}_{2}$ correlates with velocity dispersion $\sigma$ while $\langle\mathrm{Fe}\rangle:=\frac{1}{2}(\mathrm{Fe} 5270+\mathrm{Fe} 5335$ ) does not (or only weakly). Ellipticals with fine structure or peculiar core kinematics tend to have lower $\mathrm{Mg}_{2}$ than predicted for their luminosity by the luminosity - metallicity relation (see e.g. Schweizer et al. 1990, Bender et al. 1993, Carollo \& Danziger 1994).

For a large sample of $\mathrm{E} / \mathrm{S} 0 \mathrm{~s}$ in nearby clusters Jørgensen 1997 confirms the trends for field ellipticals and shows that $\mathrm{Mg}_{2}$ and $\langle\mathrm{Fe}\rangle$ both correlate with projected cluster surface density and that $\mathrm{Mg}_{2}$ strongly correlates with $\mathrm{M} / \mathrm{L}$ while $\langle\mathrm{Fe}\rangle$ does so only weakly.

Comparing the relative strengths of Fe5270, Fe5335, and $\mathrm{Mg}$ indices measured on CSPs with those observed in Galactic reference stars generally leads to the conclusion that magnesium seems to be enhanced with respect to iron in several cases. Typical values quoted for the nuclei of massive boxy Ellipticals are $[\mathrm{Mg} / \mathrm{Fe}] \sim 0.4-0.6$, while $[\mathrm{Mg} / \mathrm{Fe}] \sim 0$ for the lower luminosity disky ellipticals. $[\mathrm{Mg} / \mathrm{Fe}]$ seems to increase with increasing velocity dispersion, luminosity, metallicity, and - for cluster ellipticals - with increasing cluster surface density, while inside galaxies $[\mathrm{Mg} / \mathrm{Fe}]$ seems to be constant (see Bender 1997 for an extensive review). For Bulges the situation is less clear. While Sadler et al. 1996 find $[\mathrm{Mg} / \mathrm{Fe}] \sim 0$ for $[\mathrm{Fe} / \mathrm{H}] \geq 0$ and $[\mathrm{Mg} / \mathrm{Fe}] \sim+0.2$ for $[\mathrm{Fe} / \mathrm{H}]<0$, Jablonka et al. 1996 report a range of $[\mathrm{Mg} / \mathrm{Fe}]$ values from 0 to +0.5 with no difference between low and high luminosity bulges. Spheroidal Galaxies have $[\mathrm{Mg} / \mathrm{Fe}] \sim 0$ (Gorgas et al. 1997), the centers of luminous Sos resemble ellipticals with $[\mathrm{Mg} / \mathrm{Fe}]>0$ (Fisher et al. 1996).

The Standard Interpretation of these results relies on the assumptions that

1. relative strengths of absorption indices reflect element abundance ratios in the stellar population and

2. that these stellar element ratios reflect abundance ratios in the ISM out of which the stars were formed. 
If, and only if, both assumptions were correct (I'll show below that this is not the case for CSPs), an observed stellar $[\mathrm{Mg} / \mathrm{Fe}]_{*}>0$ would reflect an ISM abundance ratio $[\mathrm{Mg} / \mathrm{Fe}]_{\text {ISM }}>0$ at the birth of (the bulk of) the stellar population.

$\mathrm{Mg}$, like other $\alpha$-elements, is a typical SNII product that is returned to the ISM with the death of the first massive stars on a timescale of $10^{6} \mathrm{yr}$. Fe has significant $(Z 50 \%)$ contributions from SNIa, it is restored to the ISM on a timescale of several $10^{8} \mathrm{yr}$ (see e.g. Matteucci 1994).

$[\mathrm{Mg} / \mathrm{Fe}]_{\mathrm{ISM}}>0$ would have either (or several) of the following implications for the central regions of galaxies:

- an IMF favoring High Mass Star Formation (SF), at least in early evolutionary stages or for a stellar Pop3

- a characteristic timescale for SF $t_{*}<t_{\mathrm{SNI}} \sim 3 \cdot 10^{8} \mathrm{yr}$

- a lower fraction of SNI progenitor binary stars

- mass segregation and incomplete mixing (since only nuclear regions are observed)

- low metallicity stellar yields (favour $\mathrm{Mg}$ over Fe for SNII)

Whatever effect is invoked, it must be stronger in massive Es, S0s (and possibly bulges) than in low mass systems (see e.g. Faber et al. 1992, Worthey et al. 1992, Matteucci 1994, Bender et al. 1993, Thomas et al. 1997 in prep). The SF timescale $t_{*} \sim 3 \cdot 10^{8} \mathrm{yr}$ is much shorter than the collapse time of order $\sim 1 \mathrm{Gyr}$ in the classical initial collapse SF scenario for ellipticals (Larson 1974), it reminds of the duration of starbursts in spiral-spiral mergers or of the lifetime of the Infrared Ultraluminous phase. Intriguingly, ASCA measurements seem to imply $[\mathrm{Mg} / \mathrm{Fe}]>0$ for the hot ICM in galaxy clusters despite the fact that the timescale for ICM enrichment is of the order of a Hubble time.

Stellar abundance ratios in CSPs depend on the SF history, the IMF, any eventual pre-enrichment (e.g. Pop3, halo $\rightarrow$ disk) or dilution (metal-poor infall) of the ISM before the birth of the stars dominating the light at the index wavelengths today and they also depend on possible modifications inside the stars through nucleosynthesis and mixing. Clearly, abundance ratios of the sun or solar neighborhood reference stars reflect the local SF history, IMF, and all possible pre-enrichment and dilution effects of the local ISM. A serious complication comes from the fact that e.g. the iron index $\langle\mathrm{Fe}$ ) is as sensitive to iron as it is to global metallicity (which is dominated by the SNII product oxygen), as shown by Tripicco \& Bell 1995.

\section{Chemically Consistent Modelling of Galaxy Evolution}

Starting from a gas cloud with primordial abundances and specifying a SF rate (as a function of time or current gas content) and an IMF - the two fundamental parameters of our model successive generations of stars are formed with metallicities increasing from $Z=0$ to some present value. Their distribution over the HRD is calculated from stellar evolutionary tracks for various metallicities. Colour and absorption index calibrations in terms of $\left(\mathrm{T}_{\mathrm{eff}}, \log \mathrm{g},[\mathrm{Fe} / \mathrm{H}]\right)$ and a library of model atmosphere spectra for various metallicities are used to describe the photometric and spectral evolution in a chemically consistent way (Einsel et al. 1995, Möller et al. 1997). Stellar yields are taken from nucleosynthesis models for a variety of metallicities. Solving a modified form of Tinsley's equations including SNI contributions from carbon deflagration white dwarf binaries in the way prescribed by Matteucci 1994 we obtain the time evolution of ISM abundances and abundance ratios for a series of elements. We properly account for the finite lifetime of each star, i.e. we do not use an Instantaneous Recycling Approximation. The SF history appropriate for the type of galaxy together with the IMF determine the evolution of ISM abundances and abundance ratios, the age and metallicity distributions, and the colour and index evolution of a composite stellar population.

While by now the first reasonably complete sets of stellar evolutionary tracks, yields and model atmospheres for various metallicities are becoming available, the influence of non-solar abundance ratios is still far from being explored. A few recent efforts include Barbuy 1994 and Barbuy et al. 1995 who take the relative proportions of stars in various evolutionary stages from observed CMDs of globular clusters with various $[\mathrm{Mg} / \mathrm{Fe}]$ and combine with Kurucz's model atmospheres (only available for $[\mathrm{Mg} / \mathrm{Fe}]=0)$. Borges et al. 1995 give calibrations for $\mathrm{Mg}_{2}$ and $\mathrm{NaD}$ in terms of $\left(\mathrm{T}_{\text {eff }}\right.$, $\log \mathrm{g},[\mathrm{Fe} / \mathrm{H}]$, and $[\mathrm{Mg} / \mathrm{Fe}])$, and Weiss et al. 1995 show that for a $0.9 \mathrm{M}_{\odot}$ star the transition from an initial composition with $[\mathrm{Mg} / \mathrm{Fe}]=0$ to $[\mathrm{Mg} / \mathrm{Fe}]=+0.4$ increases its $\mathrm{Mg}_{2}$ index at fixed metallicity by as little as 0.03 . 
No complete grid in stellar mass, $[\mathrm{Mg} / \mathrm{Fe}]$, or $[\alpha / \mathrm{Fe}]$ is available, neither for stellar evolutionary tracks, nor for yields, colour or index calibrations, not to mention model atmospheres.

A problem with stellar yields for non-solar metallicities is that they depend on several poorly known quantities, as e.g. $\Delta Y / \Delta Z$, explosion energies, mass loss rates, remnant masses, etc., and so, might not be very reliable yet. Beyond the discrepancies between various authors there is agreement, however, that for type II SNe the ratio of the $\mathrm{Mg}$ and the Fe yields, integrated over any plausible IMF, increases considerably with decreasing metallicity. Chemically consistent modelling of the ISM abundance evolution thus results in higher $[\mathrm{Mg} / \mathrm{Fe}]_{\text {ISM }}$ ratios in early evolutionary phases as compared to models using solar metallicity yields only. If the bulk of stars in an elliptical were formed on a short timescale we would expect them to have an enhanced $\mathrm{Mg}$-to-Fe ratio frozen in. If this were then reflected in the stellar absorption line ratio of its CSP is an open question (see caveats below and also Tantalo et al. 1997). In a spiral-spiral merger scenario for the formation of an E/SO galaxy including a strong burst of SF the low metallicity stars of the gas-rich late-type spiral progenitors are all expected to have incorporated $[\mathrm{Mg} / \mathrm{Fe}]_{\mathrm{ISM}}>0$ and the stars forming in the burst on a timescale of $\sim 10^{8} \mathrm{yr}$ will have built in the rapidly increasing $[\mathrm{Mg} / \mathrm{Fe}]_{\mathrm{ISM}}$ during the burst (Fritze - v. A. \& Gerhard 1994a). This effect is seen in the spectra of young star clusters (SSPs) in the merger remnant NGC 7252 (Schweizer \& Seitzer 1993, Fritze - v. A. \& Burkert 1995).

With appropriate SF histories our models for the evolution of ISM abundances and abundance ratios were used with solar metallicity yields to interprete the redshift evolution of MgII- and CIVQSO absorption line systems that originate in galaxy haloes (Fritze - v. A. et al. 1989, 1991) and of Damped Ly $\alpha$ Absorbers (= DLAs) presumed to be (proto-)galactic disks (Fritze - v. A. \& Fricke 1995). First results from our chemically consistent models in comparison to DLA abundances are presented by Lindner et al. 1998.

\section{Results for Absorption Indices}

For SSPs of various metallicities we obtain the time evolution of colours, luminosities and indices. Using colours together with absorption indices allows to largely disentangle the age-metallicity degeneracy, since - as first shown by Worthey 94 - each colour and each index has its specific sensitivities to age and metallicity (Fritze -v. A. \& Burkert 1995). Model results include theoretical calibrations of indices, as e.g. $\mathrm{Mg}_{2}$, in terms of $[\mathrm{Fe} / \mathrm{H}]$ (Kurth et al. 1997, in prep.).

For the CSP of an elliptical galaxy, our model e.g. for a "classical L* - Elliptical" (1 zone, no dynamics with a characteristic timescale for $S F t_{*}=1 \mathrm{Gyr}$ as indicated for an initial collapse scenario) gives a broad metallicity distribution with a luminosity-weighted mean metallicity in the $\mathrm{V}$-band $\langle\mathrm{Z}\rangle_{\mathrm{V}} \sim \frac{1}{2} \cdot \mathrm{Z}_{\odot}$. While the metallicity distribution of our global galaxy model falls off sharply towards higher than solar metallicities it has a broad wing towards metallicities lower than $\frac{1}{2} \cdot Z_{\odot}$ (Möller et al. 1997). Similar results from independent modelling approaches are reported by Greggio 1997 and Arimoto 1997 (this Joint Discussion). The stellar metallicity you see depends on the wavelength of your observation. For an elliptical, it is higher by $\sim 40 \%$ in $\mathrm{K}$ than it is in the V-band. Needless to say, luminosity weighted mean stellar metallicities in general differ from the ISM metallicity. Taking a consistent metallicity distribution into account, our model reaches the observed range of $[\mathrm{MgFe}]_{*}:=\log \sqrt{\mathrm{Mgb} \cdot\langle\mathrm{Fe}\rangle} \sim 0.4-0.6$ (see e.g. Gonzalez 1993) without $[\mathrm{Mg} / \mathrm{Fe}]>0$, neither for stellar tracks, nor for index calibrations (Möller et al. 1997). With stellar evolutionary tracks from the Padova group, our chemically consistent "classical elliptical galaxy" model reaches $\mathrm{Mg}_{2} \geq 0.30$ and $\mathrm{Fe} 5335 \geq 3.1$ at ages $\geq 15 \mathrm{Gyr}$. Typical values observed in the nuclei of $\mathrm{E} / \mathrm{S} 0$ galaxies are in the range $0.18 \leq \mathrm{Mg}_{2} \leq 0.37$ and $2.0 \leq$ Fe5335 $\leq 3.5$ (Worthey et al. 1992, Gonzalez 1993). In comparison with observational data it should be kept in mind that those always refer to central regions of galaxies $\left(R<\left(\frac{1}{2}-1\right) \cdot R_{e}\right)$ and are thus expected to give upper limits only to our global galaxy models.

Increasing the timescale for $\mathrm{SF}$ we find that all models with $\mathrm{t}_{*} \leq 5 \mathrm{Gyr}$ and an average metallicity $\langle\mathrm{Z}\rangle_{\mathrm{V}}>\frac{1}{5} \cdot \mathrm{Z}_{\odot}$ reach the observed range of $[\mathrm{MgFe}]_{*} \sim 0.4-0.6$. Models with $\mathrm{t}_{*}=5 \mathrm{Gyr}$ and $\langle\mathrm{Z}\rangle_{\mathrm{V}} \geq \frac{1}{2} \cdot \mathrm{Z}_{\odot}$ reach $\mathrm{Mg}_{2} \geq 0.30$ and Fe5335 $\geq 2.7$ at ages $\geq 15 \mathrm{Gyr}$.

Revisiting our spiral-spiral merger models with interaction-triggered starbursts of various strengths (Fritze -v. A. \& Gerhard 1995a,b) it turns out that in case of a strong burst (like the one in NGC 7252) merger remnants reach the observed [MgFe]* $\sim 0.4-0.6$ about $2 \mathrm{Gyr}$ after the burst for $\langle Z\rangle_{\mathrm{V}}=\mathrm{Z}_{\odot}$ and about $4 \mathrm{Gyr}$ after the burst for $\langle\mathrm{Z}\rangle_{\mathrm{V}}=\frac{1}{2} \cdot \mathrm{Z}_{\odot}$. Merger remnants keep lower $\mathrm{Mg}_{2}$ 
and $\mathrm{Mgb}$ than ellipticals at fixed $\mathrm{M}_{\mathrm{B}}$ for about $3 \mathrm{Gyr}$ in agreement with observations (Schweizer et al. 1990, Bender et al. 1993) but can reach even the highest $\mathrm{Mg}_{2}$ and $\mathrm{Fe}$ index values observed in ellipticals about $4-5 \mathrm{Gyr}$ after the burst.

Spirals with SF truncation (via tidal stripping, sweeping or harassment) but without a burst which dynamically might transform into spheroidal galaxies - reach the observed $[\mathrm{MgFe}]_{*} \sim 0.4$ 0.6 , rapidly for $\langle Z\rangle_{\mathrm{V}}=\mathrm{Z}_{\odot}$, i.e. early type spirals, but hardly for $\langle\mathrm{Z}\rangle_{\mathrm{V}}=\frac{1}{2} \cdot \mathrm{Z}_{\odot}$ (Sbc or later). They feature average elliptical galaxy values for $\mathrm{Mg}_{2}$ and Fe5335 in case of early type spirals but values close to the lower limit of ellipticals (as appropriate for their low luminosity) in case of late type spirals (Fritze - v. A. 1997, in prep.).

All this is readily understood looking at the Lick calibrations (Gorgas et al. 1993). They directly show that $[\mathrm{MgFe}]_{*} \gtrsim 0.4$ for giants later than $\mathrm{K} 0\left(\frac{1}{2} \cdot \mathrm{Z}_{\odot}\right)$ or later than $\mathrm{G6}\left(\mathrm{Z}_{\odot}\right)$, so that whenever these stars dominate the light $[\mathrm{MgFe}]_{*}$ - values like those observed in giant Ellipticals are to be expected.

\section{Conclusions}

In addition to giving a very brief overview on observations and current interpretations of abundances and abundance ratios in composite stellar populations I stressed that elliptical galaxies are really composite in terms of stellar metallicities (and ages) and require a chemically consistent description that takes into account the range of initial stellar abundances. I showed that while for stars or SSPs, like star clusters, absorption indices do reflect ISM abundance ratios at birth, this is not the case for CSPs. Conclusions concerning the timescale for $\mathrm{SF}$ or the IMF based on $\mathrm{Mg}_{2}$ and $\mathrm{Fe}$ index data for elliptical galaxies or bulges may well be true, but need not.

\section{Acknowledgements}

I greatfully acknowledge a travel grant (Fr 916/4-1) from the Deutsche Forschungsgemeinschaft.

\section{References}

Barbuy, B., 1994, ApJ, 430, 218

Barbuy, B., de Freitas Pacheo, J. A., Idiart, T., 1995, IAU Symp. 171, p. 340

Bender, R, 1997, in The Nature of Elliptical Galaxies, $2^{\text {nd }}$ Mt. Stromly Symp. in press

Bender, R., Burstein, D., Faber, S. M., 1993, ApJ, 411, 153

Borges, A. C., Idiart, T. P., de Freitas Pacheco, J. A., Thevenin, F., 1995, AJ, 110, 2408

Carollo, C.. M., Danziger, I. J., 1994, MNRAS, 270, $523+743$

Davies, R. L., Sadler, E. M., Peletier, R. F., 1993, MNRAS, 262, 650

Einsel, C., Fritze - v. Alvensleben, U., Kruger, H., Fricke, K. J., 1995, $A \& A, 296,347$

Faber, S. M., Worthey, G., Gonzalez, J. J., 1992, IAU Symp. 149, 255

Fisher, D., Franx, M., Illingworth, G., 1996, ApJ, 459, 110

Fritz* - v. Alvensleben, U., Burkert, A., 1995, $A \& A, 300,58$

Fritz : - v. Alvensleben, U., Fricke, K. J., 1995, IAU Symp. 164, p. 457

Fritz: - v. Alvensleben, U., Gerhard, O. E., 1994, $A \mathcal{E} A, \mathbf{2 8 5}, 751+775$

Fritze - v. Alvensleben, Kruger, H., Fricke, K. J., Loose, H.-H., 1989, $A \& A, 224$, L1

Fritze - v. Alvensleben, Krulger, H., Fricke, K. J., 1991, A\&A, 246, L59

Gonzalez, J. J., 1993, PhD Thesis, Univ. of California, Santa Cruz

Gorgas, J., Faber, S. M., Burstein, D., Gonzalez, J. J., Courteau, S., Prosser, C., 1993, ApJS, 86, 153

Gorgas, J., Pedraz, S., Guzman, R., Cardiel, N., Gonzalez, J. J., 1997, ApJ, 481, L19

Greggio, L., 1997, MNRAS, 285, 151

Jablonka, P., Martin, P., Arimoto, N., 1996, $A J, 112,1415$

Jørgensen, I., 1997, MNRAS, 288, 161

Larson, R. B., 1974, MNRAS, 166, 585

Lindner, U., Fritze - v. Alvensleben, Fricke, K. J., 1998, in 13th IAP Colloquium Structure and Evolution of the IGM from QSO Absorption Line Systems, eds. P. Petitjean, S. Charlot, in press

Matteucci, F., 1994, in Panchromatic View of Galaxies, eds. Hensler et al., Editions Frontières, p. 277

Moller, C. S., Fritze - v. Alvensleben, U., Fricke, K. J., 1997, A\&A, 317, 676

Sadler, E. M., Rich, R. M., Terndrup, D. M., 1996, $A J, 112,171$

Schweizer, F., Seitzer, P., 1993, ApJ, 417, L29

Schweizer, F., Seitzer, P., Faber, S.M., Burstein, D., Dalle Ore, C.M., Gonzalez, J.J., 1990, ApJ, 364, L33,

Tantalo, R., Bressan, A., Chiosi, C., 1997, astro-ph/9705060 + 9710101

Tripicco, M. J., Bell, R. A., 1995, AJ, 110, 3035

Weiss, A., Peletier, R. F., Matteucci, F., 1995, $A \& A, 296,73$

Worthey, G., 1994, ApJS, 85, 107

Worthey, G., Faber, S. M., Gonzalez, J. J., 1992, ApJ, 398, 69 